\title{
AUTOMORPHISM GROUPS OF RULED FUNCTION FIELDS AND A PROBLEM OF ZARISKI
}

\author{
JAMES K. DEVENEY
}

\begin{abstract}
Let $K_{1}$ and $K_{2}$ be finitely generated extensions of a field $K$ and let $x$ be transcendental over $K_{1}$ and $K_{2}$, and assume $K_{1}(x)=K_{2}(x)$. The main results show that if $K$ is infinite and the group of automorphisms of $K_{2}$ over $K$ is finite, or if $K$ is finite and the group of automorphisms of $\bar{K} K_{2}$ over $\bar{K}(\bar{K}$ the algebraic closure of $K$ ) is finite, then $K_{1}$ equals $K_{2}$.
\end{abstract}

Let $K_{1}$ and $K_{2}$ be finitely generated extensions of a field $K$ and let $x_{i}$ be transcendental over $K_{i}, i=1,2$. The Zariski problem [4] asks if $K_{1}\left(x_{1}\right)=K_{2}\left(x_{2}\right)$ must $K_{1}$ and $K_{2}$ be $K$-isomorphic. Some special cases of this problem have been solved $[\mathbf{1}, \mathbf{4}]$, but in general the problem is open. In this paper we improve some known results and establish an affirmative answer for a new class of fields in a special case.

Definition 1. Let $L$ be a finitely generated extension of a field $K$. If $K$ is infinite and the group of $K$-automorphisms of $L$ is finite, $\mid$ aut ${ }_{K} L \mid<\infty$, then $L$ is of general type over $K$. If $K$ is finite and $\mid$ aut $_{K} L \bar{K} \mid<\infty$ for $\bar{K}$ an algebraic closure of $K$, then $L$ is of general type over $K$.

The motivation for this definition is the paper of Husemoller [3]. He discusses the canonical dimension of a variety over an algebraically closed field. He defines an $r$-dimensional variety $V$ to be of general type provided the canonical dimension of $V$ is $r$ (which for example, includes curves of genus greater than 1). He then goes on to show that if $k(v)$ is the function field of a variety of general type, then the group of $k$-automorphisms of $k(v)$ is finite. The special definition for $K$ finite is to prevent $K(x)$ from being of general type over $K$. The main results of this paper related to the Zariski problem assert that if $K_{2}$ is of general type over $K$, and $x_{1}=x_{2}$, then $K_{1}$ equals $K_{2}$. For the case of an infinite base field $K$, Samuel [4] has shown $K_{1}$ and $K_{2}$ are $K$-isomorphic. For the case of a finite base field, nothing had been known.

We first make a few general observations. In order to achieve an affirmative answer to the Zariski problem, one can assume $K_{1} \cap K_{2}=K$. Thus one can assume $K$ is algebraically closed in $K_{1}\left(x_{1}\right)$. Furthermore, since $K_{1}\left(x_{1}\right)$ is separable over $K_{1}$ and $K_{2}$, it is separable over their intersection [2, Theorem 1.1, p. 1304], and hence each $K_{i}$ is separable over $K$, i.e. is regular over $K$. We note that if $\bar{K}$ denotes the algebraic closure of $K$, and $K_{2}$ is regular over $K$, then every $K$-automorphism of $K_{2}$

Received by the editors April 19, 1983 and, in revised form, August 1, 1983.

1980 Mathematics Subject Classification. Primary 14J25; Secondary 12F20.

Key words and phrases. Function field, automorphisms. 
has a unique extension to a $\bar{K}$-automorphisms of $K_{2} \bar{K}=K_{2} \otimes_{K} \bar{K}$. Thus if $K_{2} \bar{K}$ is the function field of a variety of general type over $\bar{K}$, and $K_{2}$ is regular over $K, K_{2}$ is of general type over $K$. Part of the proof of Theorem 2 is essentially due to Roquette [5, Lemma 1, p. 209].

Theorem 2. Assume $L=K_{1}(x)=K_{2}(x) \supset K$ where $x$ is transcendental over $K_{i}$, and $K_{i}$ is a finitely generated extension of an infinite field $K, i=1,2$. If the group of $K$-automorphisms of $K_{2}$ is finite, then $K_{1}=K_{2}$.

Proof. Let $\left\{w_{1}, w_{2}, \ldots, w_{r}\right\}$ generate $K_{2}$ over $K$. Each of these is a rational function $f_{i}(x) / g_{i}(x)$ in $x$ with coefficients in $K_{1}$. Let the nonzero coefficients of $f_{i}(x)$ be $\left\{a_{i j}\right\}$ and the nonzero coefficients of $g_{i}(x)$ be $\left\{b_{i j}\right\}$. Let $a_{i}=f_{i}(x), b_{i}=g_{i}(x)$. Each of the elements of $\left\{a_{i}, a_{i j}, b_{i}, b_{i j}\right\}$ is also a rational function in $x$ with coefficients in $K_{2}$. There are only a finite number of prime divisors of $K_{2}(x)$ over $K_{2}$ for which the associated place is either 0 or $\infty$ at any given element. Since $|K|=\infty$, there is an infinite number of elements $\alpha_{i} \in K$ such that the $\left(x-\alpha_{i}\right)$-place of $K_{2}(x)$ onto $K_{2}$ is finite and nonzero at each $\left\{a_{i}, a_{i j}, b_{i}, b_{i j}\right\}$. Thus, for each of these places $p_{\left(x-\alpha_{i}\right)}$

$$
p_{\left(x-\alpha_{l}\right)\left(w_{j}\right)}=p_{\left(x-\alpha_{i}\right)}\left(\frac{f_{j}(x)}{g_{j}(x)}\right)=\frac{p_{\left(x-\alpha_{i}\right)}\left(f_{j}\right)\left(\alpha_{i}\right)}{p_{\left(x-\alpha_{i}\right)}\left(g_{j}\right)\left(\alpha_{i}\right)} \in p_{x-\alpha_{i}}\left(K_{l}\right) .
$$

Thus $K_{2} \subseteq p_{x-\alpha_{i}}\left(K_{1}\right)$, i.e. $K_{2}=p_{x-\alpha_{i}}\left(K_{1}\right)$. Thus we have an infinite number of elements $\left\{\alpha_{i}\right\}$ of $K$ such that the $p_{x-\alpha_{i}}$ place of $K_{2}(x)$ over $K_{2}$ gives a $K$-isomorphism of $K_{1}$ onto $K_{2}$. Symmetrically, we can certainly get a single $K$-isomorphism $\sigma$ : $K_{2} \rightarrow K_{1}$.

We now assume there exists an element, $z$, of $K_{1}$ which is not an element of $K_{2}$ and we get a contradiction. $z=r(x)$ is a nonconstant rational function in $x$ with coefficients in $K_{2}$. Choose $\alpha_{0} \in\left\{\alpha_{i}\right\}$ as above and consider $r(x)-r\left(\alpha_{0}\right)$, which is also a nonconstant rational function with coefficients in $K_{2}$. As noted above, each $p_{\left(x-\alpha_{i}\right)}$ defines a $K$-isomorphism of $K_{1}$ onto $K_{2}$. Call this isomorphism $\bar{\alpha}_{i}$. Then each $\bar{\alpha}_{i} \circ \sigma$ defines a $K$-automorphism of $K_{2}$. Since the group of $K$-automorphisms of $K_{2}$ is finite, and $\bar{\alpha}_{i} \circ \sigma=\bar{\alpha}_{j} \circ \sigma$ if and only if the isomorphisms $\bar{\alpha}_{i}=\bar{\alpha}_{j}$, there must be some infinite family of automorphisms $\bar{\alpha}_{i}$ which are equal. We may assume $\alpha_{0}$ is in this family. But then each of the elements associated to the automorphisms must be a root of the nonzero rational function $r(x)-r\left(\alpha_{0}\right)$. But this is a contradiction since a nonzero rational function has only a finite number of roots. Thus $K_{1}=K_{2}$.

Let $K$ be a field and let $\{x, y\}$ be algebraically independent over $K$. Note that $K(y)(x)=K(y-x)(x)$, and yet $K(y) \neq K(y-x)$. Moreover, if $K$ is finite, $\mid$ aut $_{K} K(y-x) \mid<\infty$. Thus neither of the assumptions in the theorem is superfluous.

Corollary 3. Assume $K$ is infinite, the group of $K$-automorphisms of $K_{1}$ is finite and $x$ is transcendental over $K_{1}$. Then the natural injection $\sigma$ : aut ${ }_{K} K_{1} \rightarrow$ aut $_{K(x)} K_{1}(x)$ is also surjective. 
Proof. Clearly every $K$-automorphism $\theta$ of $K_{1}$ can be uniquely extended to a $K(x)$-automorphism of $K_{1}(x)$ by defining $\theta(x)=x$. Now let $\theta$ be any $K(x)$-automorphism of $K_{1}(x)$. Then $K_{1}(x)=K_{1}^{\theta}(\theta(x))=K_{1}^{\theta}(x)$, where $K_{1}^{\theta}$ denotes the image of $K_{1}$ under $\theta$. By Theorem $2, K_{1}=K_{1}^{\theta}$, i.e. $\theta$ is an extension of a $K$-automorphism of $K_{1}$.

For $K(y)$ a simple transcendental extension of $K$, the $K(x)$-automorphism of $K(y, x)$ which sends $y$ to $y+x$ will not be the extension of any $K$-automorphism of $K(y)$.

Lemma 4. Let $K_{1}$ and $K_{2}$ be subfields of a field $L$ and assume $K_{1} \cap K_{2}=K$. If $L$ and $F$ are linearly disjoint over $K$, and $K_{1} F=K_{2} F$, then $K_{1}=K_{2}$.

Proof. By the standard lemma on linear disjointness, $K_{i} F$ and $L$ are linearly disjoint over $K_{i}$, and hence $K_{i} F \cap L=K_{i}, i=1,2$. Thus if $K_{1} F=K_{2} F, K_{1}=K_{2}$.

THEOREM 5. Assume we have $L=K_{1}(x)=K_{2}(x) \supset K$ where $x$ is transcendental over $K_{i}$, and $K_{i}$ is a finitely generated extension of a finite field $K$. If $K_{2}$ is of general type over $K$, then $K_{1}=K_{2}$.

Proof. If $K_{1} \cap K_{2}$ is not a finite field, then we may apply Theorem 2. Thus we may assume $K_{1} \cap K_{2}=K$, and hence $K$ is algebraically closed in $K_{1}(x)$. Since $K$ is perfect, $K_{1}(x)$ is regular over $K$. Thus $K_{1}(x)$ is linearly disjoint over $K$ from $\bar{K}$, the algebraic closure of $K$. Since $K_{2} / K$ is of general type, Theorem 2 asserts $K_{1} \bar{K}=K_{2} \bar{K}$. By Lemma $4, K_{1}=K_{2}$.

It should be noted that Theorem 5 is true under slightly more general conditions. For example, if $\mid$ aut $_{K(y)} K_{2}(y) \mid<\infty$, where $y$ is transcendental over $K_{2}(x)$, then a similar application of Theorem 2 and Lemma 4 shows $K_{1}=K_{2}$.

The main results of this paper are related to [4, Theorem 2, p. 87 and Corollary 2 , p. 88]. Nagata uses the hypothesis (N) that no algebraic extension of $K_{2}$ is ruled over $K$, whereas the present paper uses the hypothesis (D) that $\left|\mathrm{Aut}_{K}\left(K_{2}\right)\right|<\infty$. Consider the 1-dimensional case, i.e., tr.d. $\left(K_{2} / K\right)=1$. For $K$ of characteristic 0 , the fields satisfying (D) are exactly those of genus $\geqslant 2$, while those satisfying $(N)$ are exactly those of genus $\geqslant 1$. The latter point follows since a separable base charge cannot lower the genus. Thus in this case Nagata's theorem implies the present result. If the characteristic of $K$ is $p \neq 0$, then there exist examples of curves of genus $\geqslant 2$ such that base charge drops the genus to 0 , e.g. let $y^{2}=x^{p}-a$, $a^{1 / p} \notin K$, and adjoint $a^{1 / p}$ to $K(x, y)$. For these curves, (N) does not hold but (D) does. The author is indebted to the referee for the above comments.

\section{REFERENCES}

1. J. Deveney, Ruled function fields, Proc. Amer. Math. Soc. 86 (1982), 213-215.

2. J. Deveney and J. Mordeson, Subfields and invariants of inseparable field extensions, Canad. J. Math. 29 (1977), 1304-1311.

3. D. Husemoller, Finite automorphism groups of algebraic varieties, Proc. Sympos. Pure Math., vol. 37. Amer. Math. Soc., Providence, R. I., 1980, pp. 611-619.

4. M. Nagata, $A$ theorem on valuation rings and its applications, Nagoya Math. J. 29 (1967), 85-91.

5. P. Roquette, Isomorphisms of generic splitting fields of simple algebras, J. Reine Angew. Math. 214-215 (1964), 207-226.

Department of Mathematical Sciences, Virginia Commonwealth University, Richmond, VIRGINIA 23284 